\title{
Monte Carlo Integration Technique for Method of Moments Solution of EFIE in Scattering Problems
}

\author{
Mrinal MISHRA, Nisha GUPTA \\ Department of Electronics and Communication Engineering, Birla Institute of Technology, Mesra, India \\ Email: mrinal.mishra@gmail.com,ngupta@bitmesra.ac.in
}

Received May $8^{\text {th }}, 2009$; revised July $10^{\text {th }}, 2009$; accepted July $18^{\text {th }}, 2009$.

\begin{abstract}
An integration technique based on use of Monte Carlo Integration is proposed for Method of Moments solution of Electric Field Integral Equation. As an example numerical analysis is carried out for the solution of the integral equation for unknown current distribution on metallic plate structures. The entire domain polynomial basis functions are employed in the MOM formulation which leads to only small number of matrix elements thus saving significant computer time and storage. It is observed that the proposed method not only provides solution of the unknown current distribution on the surface of the metallic plates but is also capable of dealing with the problem of singularity efficiently.
\end{abstract}

Keywords: Scattering, EFIE, Method of Moments, Monte Carlo Integration

\section{Introduction}

The Method of Moments (MoM) [1] is one of the widely used numerical techniques employed for the solution of Integral Equations. The MoM is based upon the transformation of an integral equation, into a matrix equation. However, the application of the spatial-domain MoM to the solution of integral equation is quite time consuming. The matrix-fill time would be significantly improved if these integrals can be evaluated efficiently. The MoM employs expansion of the unknown function inside the integral in terms of known basis functions with unknown coefficients to be determined. Point matching technique or Galerkin's technique commonly employed in MoM results in a system of linear equations equal in number to that of unknown coefficients. This leads to a matrix equation for the coefficients. The matrix thus obtained is called the 'moment' matrix. The unknown coefficients can then be obtained by matrix inversion.

The MoM method involves two approaches, the sub domain $[2,3]$ and the entire domain $[4,5]$ approaches, essentially based on the two kinds of basis functions employed for the expansion of the unknown function on the metal surface. The entire domain basis functions extend over the whole region occupied by the structure, whereas the sub domain basis functions are defined to exist over a section of the structure and have a zero value over the rest of its portion. The choice of the type of basis function depends upon the size and shape of the metallic structure in the problem. The advantage with the sub domain basis functions is that due to their flexibility to be defined over small polygonal domains of varying sizes. The whole structure under investigation can be modeled as consisting of large number of such polygonal sub domains, thus making possible the analysis of complicated shaped structures. The disadvantage with these basis functions is that they are limited to electrically small and moderately large structures, as the number of sub domains required to model large structures accurately becomes very large. This results in the moment matrix of a large size increasing the computation costs in terms of memory and CPU time.

The entire domain basis functions, on the other hand, require a very few number of expansion terms. These are also capable of analyzing electrically large structures and the solution obtained with these functions are more accurate than the sub domain basis functions. This results in a faster and more accurate solution, thus reducing the computational cost. One of the requirements of the entire domain basis functions is a prior knowledge of the distribution of the unknown quantity for the kind of the structure under consideration. The effectiveness of a MoM numerical solution depends on a judicious choice of basis functions. The optimal choice of the basis function is one that provides solutions with the fewest number of expansion terms and in shortest computational time. These functions should incorporate as closely as possible the physical conditions of the actual distribution of the unknown quantity on the region of interest. In this paper, entire domain polynomial basis function is utilized 
which results in the reduction of computational cost and an increase in the accuracy of the result.

The other aspect of the MoM formulation is the problem of the singularity of the function that is to be integrated to obtain the matrix elements, in both the approaches of the MoM formulation. In the point matching MoM approach using sub-domain basis functions, only a few matrix elements, whereas using entire domain approach, all the matrix elements are obtained by integration of singular functions. Various analytical and numerical techniques have been adopted to deal with such integrals. The Monte Carlo Integration (MCI) technique $[6,7]$ proposed in this paper is not only capable of solving the scattering problem but also deals efficiently with the problem of singularity.

To demonstrate the capability of the above mentioned technique, the problem is formulated in terms of an integral equation to determine the current distribution on square metallic plate. The MCI technique has been proposed to tackle scattering problem from an infinitesimally thin square metallic plate structures in the MoM formulation of the problem. The entire domain basis functions are utilized in the MoM matrix solution and hence reduce the computational cost to the great extent. Besides, it is also capable of handling the singularity aspect of the Green's function easily and more efficiently. The typical simulation demonstrates the application of the proposed technique and also validates the result against the Benchmark solution [8].

\section{Mathematical Concept}

Monte Carlo methods are useful for obtaining solutions to problems involving integration which are too complicated to be solved analytically or by other numerical methods. Standard numerical integration techniques do not work very well on high-dimensional domains, especially when the integrand is not smooth. Although the quadrature rules of integration typically work very well for one-dimensional integrals, problems occur when extending them to higher dimensions.

Monte Carlo methods have advantages over numerical methods in a space of many dimensions. Their efficiencies relative to other numerical methods increase when the dimension of the problem increases e.g. Quadrature formula becomes very complex while MCI technique remains almost unchanged in more than one dimension. In addition to this, the convergence of the MCI is independent of dimensonality regardless of the smoothness of the integrand. Monte Carlo integration is simple since only two basic operations are required, namely sampling and point evaluation. It is also suited for large structures and highly complex problems for which definite integral formulation is not obvious and standard analytical techniques are ineffective. Sampling can be used even on domains that are not well-suited to numerical quadrature.
The idea of Monte Carlo integration is to evaluate the integral using random sampling as

$$
I=\int_{\Omega} f(x) d x
$$

where $f$ is a function of vector $\boldsymbol{x}, \Omega$ is domain of integration. The Monte Carlo integration is popular for complex $f$ and/or $\Omega$. In its basic form, this is done by independently sampling $\mathrm{N}$ points $x_{1}, \ldots, x_{N}$ according to some convenient density function $p$, and then computing the estimate

$$
F_{N}=\frac{\Omega}{N} \sum_{i=1}^{N} \frac{f\left(x_{i}\right)}{p\left(x_{i}\right)}
$$

where $p\left(x_{i}\right)$ is the probability density function or pdf. Here the notation $F_{N}$ is used rather than $\boldsymbol{I}$ to emphasize that the result is approximate, and that its properties depend on how many sample points are chosen. If $p\left(x_{i}\right)$ is the uniform probability density, then the integral is simply

$$
I \cong \frac{\Omega}{N} \sum_{i=1}^{N} f\left(x_{i}\right)
$$

The MCI methods are better suited than quadrature methods for integrands with singularities. It is particularly helpful for integrand that have large values on a relatively small part of the domain due to singularities. It can be applied to handle such integrands effectively, even in situations where there is no analytic transformation available to remove the singularity. The simple way to handle the singularity using MCI is to ignore a region around the singularity and let this region become smaller as $\mathrm{N}$ increases [9-13]. In this approach the total region is split into $\mathbf{r}>\mathbf{r}_{\min }$, where the numerical integration is perform, and a small region $\mathbf{r}<\mathbf{r}_{\min }$ which is apparently left out as long as it has a small or negligible contribution for large values of $\mathrm{N}$. The event generators use therefore a cutoff $\mathbf{r}_{\min }$ to avoid this region of singularity i.e., the random points generated in MCI are restricted to fall within this excluded region. This does not require any extra effort to handle the singularity problem as the required condition can be embedded directly in the MCI technique itself in a single statement of the MATLAB code employed for the purpose in simulation.

\section{Numerical Example}

As an example, the electric field integral equation is solved by method of moments for the unknown surface current density on a square metallic plate. The plate is an infinitesimally thin $\lambda \mathrm{x} \lambda$ square in free space, with limits $-1.0 \mathrm{~m}$ to $1.0 \mathrm{~m}$ along both the $x$ and $y$ axes. The scatterer is excited normally by an incident plane wave with the electric field $\mathbf{E}^{i}$ having a magnitude $1.0 \mathrm{Vm}^{-1}$ and polarized along a scatterer edge, in this case the $y$-axis. The 


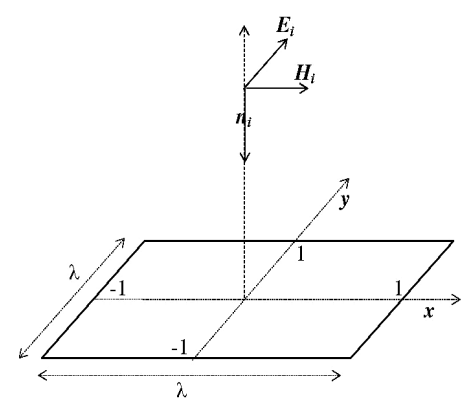

Figure 1. Geometry of the $\lambda \times \lambda$ square P. E. C. scatterer incident upon by a plane wave with $E_{i}$ polarized along the $y-$ axis, $\lambda=2 \mathrm{~m}$

geometry is shown in Figure 1.

From Maxwell's equations we can obtain vector expression for the total electric field

$$
\mathbf{E}^{t}=\mathbf{E}^{i}+\mathbf{E}^{s}
$$

where $\mathbf{E}^{t}$ is the incident field and $\mathbf{E}^{s}$ is the scattered

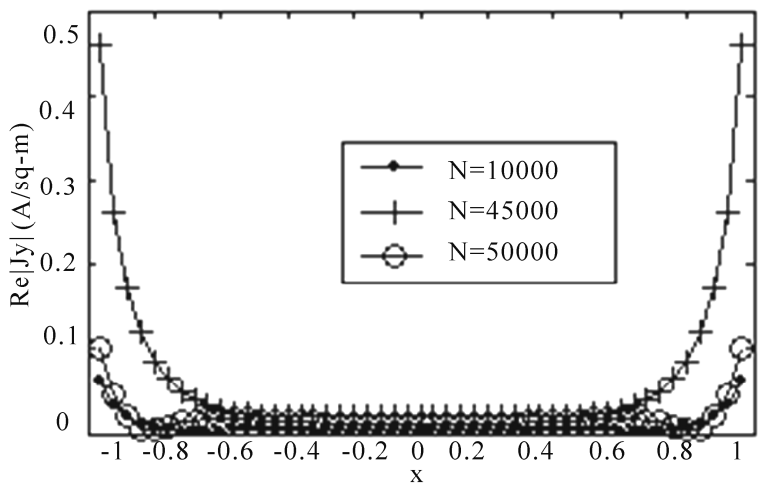

(a)

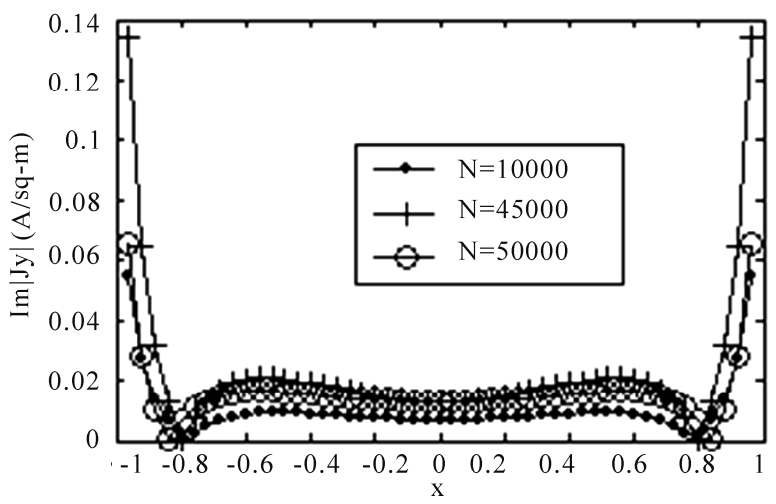

(c) field as:

$$
\mathbf{E}^{s}=\left(1 / j \omega \varepsilon_{0} \mu_{0}\right)\left[\nabla(\nabla \bullet \mathbf{A})+k^{2} \mathbf{A}\right]
$$

where

$$
\mathbf{A}(\mathbf{r})=\frac{\mu_{0}}{4 \pi} \int_{\text {conductingsurface }} \mathbf{J}\left(\mathbf{r}^{\prime}\right) \frac{e^{-j k\left|\mathbf{r}-\mathbf{r}^{\prime}\right|}}{\left|\mathbf{r}-\mathbf{r}^{\prime}\right|} d \mathbf{r}^{\prime}
$$

is the vector potential.

Applying the boundary condition $\mathbf{n} \times \mathbf{E}^{t}=0$ on the scatterer, we get the electric field integral equation (EFIE) for the unknown current density $\mathbf{J}\left(\mathbf{r}^{\prime}\right)$ :

$$
-\mathbf{E}^{i}=\mathbf{E}^{s}=\left(1 / j \omega \varepsilon_{0} \mu_{0}\right)\left[\nabla(\nabla \bullet \mathbf{A})+k^{2} \mathbf{A}\right]
$$

For method of moments (MoM) solution of the integral equation, taking some known basis function $f_{n}\left(\mathbf{r}^{\prime}\right)$, the unknown current on the conducting surface can be expanded as:

$$
\mathbf{J}\left(\mathbf{r}^{\prime}\right)=\sum_{n=1}^{M} a_{n} f_{n}\left(\mathbf{r}^{\prime}\right)
$$

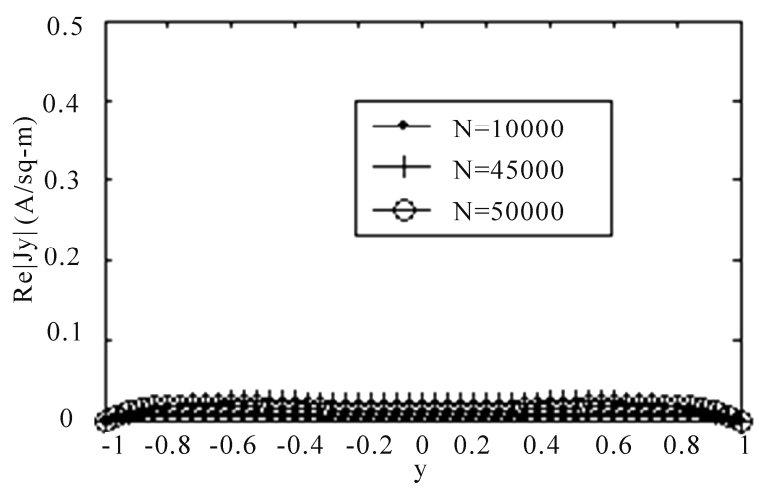

(b)

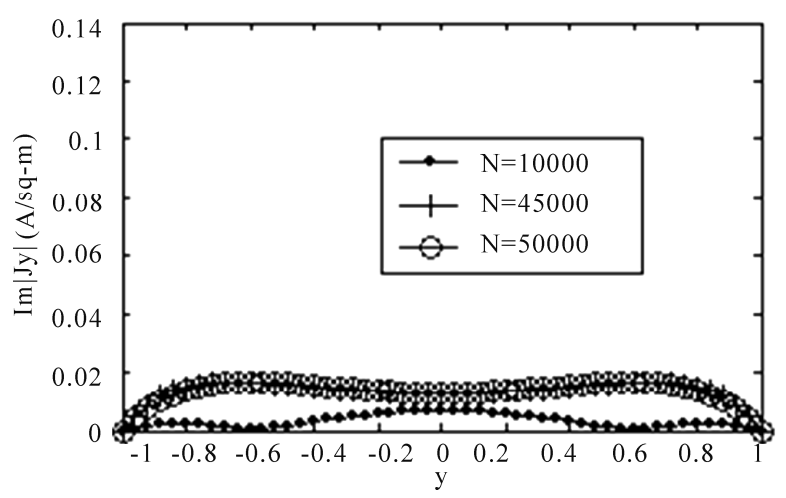

(d)

Figure 2. (a) Solution for the $J_{y}$-current component (real part) along the $X$ axis of the scatterer obtained for different number of random points generations; (b) Solution for the $J_{\mathbf{y}}$-current component (real part) along the $Y$ axis of the scatterer obtained for different number of random points generations; (c) Solution for the $J_{y}$-current component (imaginary part) along the $X$ axis of the scatterer obtained for different number of random points generations; (d) Solution for the $J_{\mathbf{y}}$-current component (imaginary part) along the $Y$ axis of the scatterer obtained for different number of random points generations 
where $a_{n} ; n=1,2, \ldots, M$ are unknown amplitudes of the basis functions and are to be determined. This expansion is applied over the same number of field points as the number of expansion terms, which transforms the integral equation into a set of simultaneous algebraic equation in the unknown coefficients, which can be written in the matrix form as:

$$
\left[Z_{m n}\right]\left[a_{n}\right]=\left[-E_{n}^{i}\right]
$$

or

$$
\left[a_{n}\right]=\left[Z_{m n}\right]^{-1}\left[-E_{n}^{i}\right]
$$

The matrix elements of the matrix equation are formed using numerical integration (such as MCI technique) of the singular function that results after differentiation of the Green's function.

The problem under investigation is an entire domain MoM problem. The entire domain basis functions for the unknown current density are the modified polynomial functions, with edge correction and symmetry considerations as stated in the benchmark solution [8] and presented as follows:

$$
\begin{aligned}
& J_{x}(x, y) \cong \sum_{\substack{i=1 \\
(2)}}^{n_{y x}}\left\{\sum_{\substack{j=3 \\
(2)}}^{n_{x x}} a_{x i j}\left(x^{j}-x\right)\right\} \cdot \frac{y^{i}}{\sqrt{1-y^{2}}} \\
& J_{y}(x, y) \cong \sum_{\substack{i=0 \\
(2)}}^{n_{x y}}\left\{\sum_{\substack{j=3 \\
(2)}}^{n_{y y}} a_{y i j}\left(y^{j}-1\right)\right\} \cdot \frac{x^{i}}{\sqrt{1-x^{2}}}
\end{aligned}
$$

where $n_{x x}=n_{y y}=8$ and $n_{x y}=n_{y x}=7$. The subscript (2) below the summation sign means that the indices $i$ and $j$ are to be increased with a step size 2 . Thus the total number of expansion terms for $x$-current is 12 and for $y$-current is 16 , thus making the total number of unknown coefficients $=28$. This leads to the formation of the Z-matrix of order $28 \times 28$, far less than the number of coefficients required in sub domain analysis, which in case of such a large scatterer becomes extremely large for accurate analysis. Though, the Galerkin's method in MoM is a suitable choice for the problem under investigation, the present formulation employs the point matching technique in MoM specifically to demonstrate the singularity aspect of integrand. The point matching technique makes it essential that all the matrix elements that are evaluated, involve integration of singular integrands (singular kernels of the integral equation). Thus it is necessary to adopt means that can take care of the singularity inside the integral and give a good approximation of the actual result. The technique adopted here is the Monte Carlo Integration (MCI) technique that overcomes the singularity, making integration much simpler and justified in case of two dimensional and three dimension-

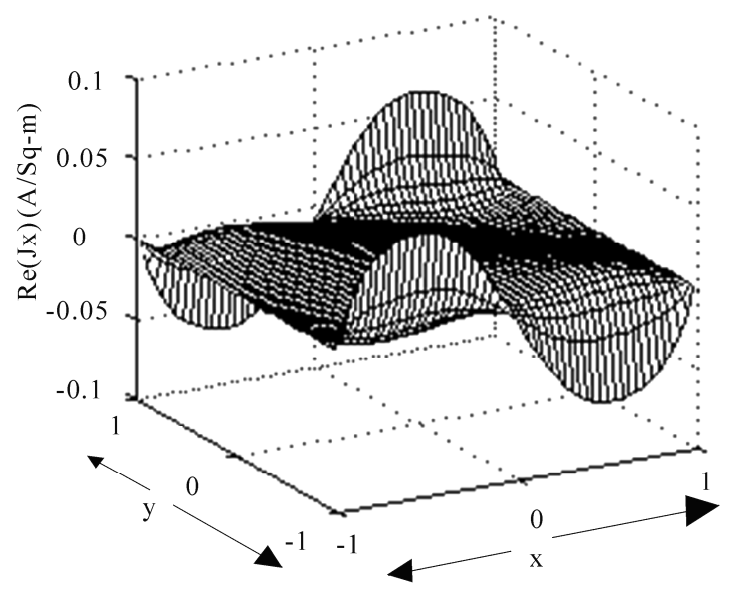

(a)

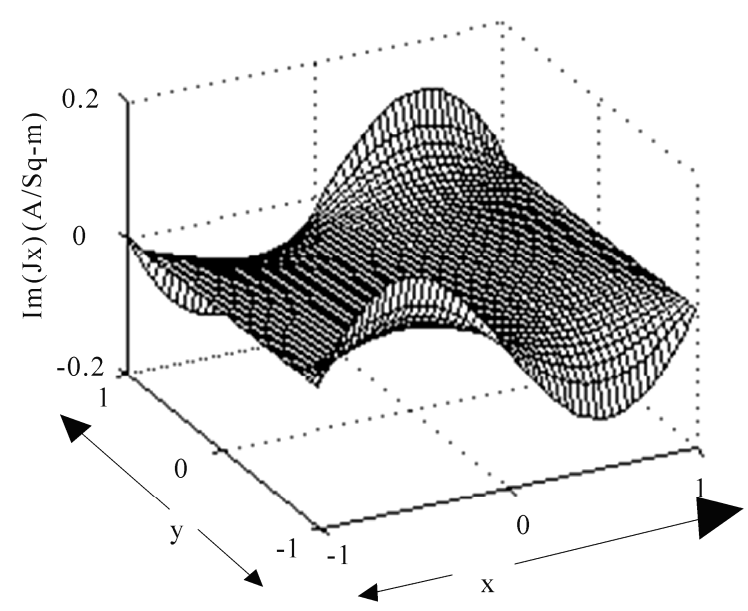

(b)

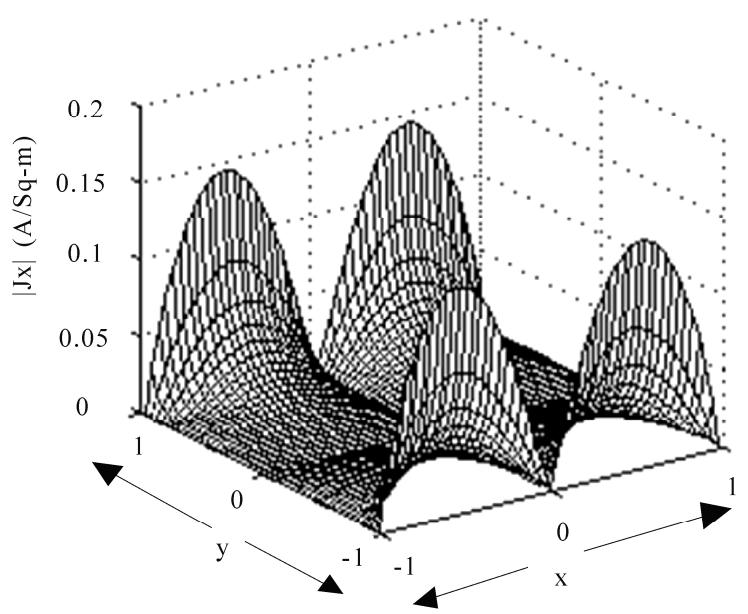

(c)

Figure 3. (a) Solution for the $J_{x}$ current component (Real part) over the scatterer; (b) Solution for the $J_{x}$ current component (Imaginary part) over the Scatterer; (c) Solution for the $J_{x}$ current component (Magnitude) over the Scatterer 
al scattering problems. The method that has dealt with the singularity here is named as the 'local correction technique'. Since MCI is based on random generation of points inside the entire domain of integration, care must taken as to prevent the points fall inside a certain region $\mathbf{r}<\mathbf{r}_{\min }$, the region of singularity.

The Figures 2(a) and 2(b) show the real part of $J_{y}$ as seen along the x-axis while the Figures 2(c) and 2(d) show the real and imaginary parts of $J_{y}$ as seen along the $\mathrm{y}$-axis, for different numbers $\mathrm{N}$ of random points taken for the MCI. The figures make evident two things. First, the results for all the values of $\mathrm{N}$ show a good agreement with the results obtained in the benchmark solution [8] using MoM for the solution. Second, the solution with $\mathrm{N}=$ 10000 points converge to that obtained for $\mathrm{N}=45000$ and $\mathrm{N}=50000$ points, stating that $\mathrm{N}=10000$ points are sufficient enough in the MCI for the problem under in vestigation, thereby decreasing the computational burden to a great extent. Figures 3(a), 3(b) and 3(c) show the results for the 3-D current distribution for the real part, imaginary part and magnitude of $J_{x}$. Total number of random points for the MCI to evaluate the elements in the impedance matrix for MoM solution has been taken to be 10,000. It is found that the results show good agreement with those obtained in benchmark solution [8].

\section{Conclusions}

The Monte Carlo integration technique in the MoM solution of the integral equations has been proposed. The technique employs the entire-domain polynomial basis functions along with Monte Carlo Integration technique in MOM solution of the problem under investigation. In the Monte Carlo Integration technique, a local correction technique is employed to deal with the singularity aspect of the kernel very efficiently and easily. For the demonstration of the present method, the current distribution is investigated on a two dimensional square plate scatterer. Where comparisons are available, it is found that this technique yields results which compare very closely to those of other methods. In addition to the rapid convergence of the Monte Carlo integral with respect to total number of random points, the small number of basis functions $(<10)$ is needed to provide accurate results. Further research is being carried out to extend the method to three dimensional structures.

\section{Acknowledgements}

This research work was supported by the Council of Sci- entific and Industrial Research (CSIR), Pusa, New Delhi, India-110012.

\section{REFERENCES}

[1] R. F. Harrington, "Field computation by moment methods," New York, Macmillan, 1968.

[2] C. A. Balanis, "Antenna theory: Analysis and design," Harper \& Row, New York, pp. 283-321. 1982.

[3] C. M. Bulter and D. R. Wilton, "Analysis of various numerical techniques applied to thin-wire scatterers," IEEE Transactions, Vol. AP-23, No. 4, pp. 524-540, 1975.

[4] B. M. Notaros and B. D. Popovic, "General entire-domain method for analysis of dielectric scatterers," IEE Proceedings - Microwaves, Antennas and Propagation, Vol. 143, No. 6, pp. 498-504, 1996.

[5] M. Djordjevic and B. M. Notaros, "Double higher order method of moments for surface integral equation modeling of metallic and dielectric antennas and scatterers," IEEE Transactions on Antennas and Propagation, Vol. 52, No. 8, pp. 2118-2129, 2004.

[6] W. H. Press, S. A. Teukolsky, W. T. Vetterling, and B. P. Flannery, "Numerical recipes, second edition," Cambridge University Press, 1992.

[7] M. N. O. Sadiku, "Numerical techniques in electromagnetics," CRC Press, New York.

[8] B. M. Kolundžija, "Accurate solution of square scatterer as benchmark for validation of electromagnetic modeling of plate structures," IEEE Transactions on Antennas and Propagation, Vol. 46, No. 7, pp. 1009-1014, 1998.

[9] T. Pillards, "Quasi-Monte Carlo integration over a simplex and the entire space," Ph. D. Thesis, Katholieke Universiteit Leuven, Belgium, ISBN 90-5682-741-3, 2006.

[10] J. Hartinger, R. F. Kainhofer, and R. F. Tichy, "QuasiMonte Carlo algorithms for unbounded, weighted integration problems," Journal of Complexity, Vol. 5, No. 20, pp. 654-668, 2004,

[11] A. B. Owen, "Quasi-Monte Carlo for integrands with point singularities at unknown locations," Monte Carlo and Quasi-Monte Carlo Methods, pp. 403-418, 2004.

[12] M. Mishra and N. Gupta, "Singularity treatment for integral equations in electromagnetic scattering using Monte Carlo integration technique," Microwave and Optical Technology Letters, Vol. 50, No. 6, pp. 1619-1623, June 2008.

[13] M. Mishra and N. Gupta, "Monte Carlo integration technique for the analysis of electromagnetic scattering from conducting surfaces," Progress In Electromagnetics Research, PIER 79, pp. 91-106, 2008. 